\title{
Caracterização de argilas calcárias utilizadas na produção de revestimentos cerâmicos no Estado de Sergipe - Brasil
}

\author{
(Characterization of calcareous clay used in the production of \\ ceramic tiles in Sergipe State - Brazil) \\ C. P. Santos ${ }^{1}$, H. A. Oliveira ${ }^{1}$, R. M. P. B. Oliveira ${ }^{2}$, Z. S. Macedo ${ }^{1}$ \\ ${ }^{1}$ Programa de Pós-graduação em Ciência e Engenharia de Materiais, \\ ${ }^{2}$ Departamento de Ciência e Engenharia de Materiais, Universidade Federal de Sergipe, S. Cristóvão, SE \\ cochiran@hotmail.com,zmacedo@ufs.br
}

\begin{abstract}
Resumo
O presente trabalho tem por objetivo caracterizar quatro argilas diferentes utilizadas na produção de revestimento cerâmico do tipo BIIb no Estado de Sergipe, Brasil. As argilas foram caracterizadas por difração de raios X, fluorescência de raios X, análise termogravimétrica, análise térmica diferencial, distribuição de tamanho de partículas, limites de Atterberg e dilatometria. A partir dos resultados obtidos foi possível detectar concentrações de calcita entre 1,1 e 13,7\%, a depender da origem da argila. Esta informação é determinante para ajustes no ciclo de queima e previsão da energia mínima para que a sinterização ocorra. Foram produzidos corpos de prova por prensagem uniaxial em matriz retangular de $120 \mathrm{~mm}$ x $56 \mathrm{~mm}$ x $6 \mathrm{~mm}$ a uma pressão de $28 \mathrm{MPa}$, os quais foram queimados a $1130^{\circ} \mathrm{C}$ com ciclo de queima de $23 \mathrm{~min}$ e patamar de $3 \mathrm{~min}$. Os corpos de prova foram caracterizados quanto à absorção de água e módulo de ruptura à flexão, e sua microestrutura foi investigada por microscopia eletrônica de varredura. Através dos resultados, foi possível confirmar a correlação entre a formação de fase líquida e redução de porosidade nos corpos cerâmicos sinterizados a 1150 ${ }^{\circ} \mathrm{C}$. As energias de ativação para o processo de sinterização foram determinadas a partir dos ensaios dilatométricos e os resultados mostraram que, à medida que se diminui o teor de calcário combinado com o aumento do teor de materiais fundentes, a sinterização ocorre em temperaturas mais baixas, resultando em menores gastos energéticos no processo de produção.
\end{abstract}

Palavras-chave: argila, revestimento cerâmico, caracterização, calcita, energia de ativação.

\begin{abstract}
This work aims to evaluate four different clays used in the production of BIIb type ceramic tiles in Sergipe State, Brazil. The clays were characterized by X-ray diffraction, X-ray fluorescence, thermogravimetric analysis, differential thermal analysis, particle size distribution, Atterberg limits and dilatometry. From the results, it was possible to detect calcite concentrations between 1.1 and $13.7 \%$ depending on the origin of the material. This information is essential for adjustments in the firing cycle and to predict the minimum energy for sintering. Ceramic bodies were formed by uniaxial pressing of 28 MPa into rectangular bodies with dimensions of $120 \mathrm{~mm} \times 56 \mathrm{~mm} \times 6 \mathrm{~mm}$. These ceramic bodies were then fired at $1130{ }^{\circ} \mathrm{C}$ using a 23 min firing cycle and 3 min of soaking time. Water absorption and modulus of rupture in bending were measured and the microstructure was investigated by electron scanning microscopy. From the results, it was possible to observe a correlation between liquid phase formation and reduction of porosity in ceramic bodies sintered at $1150{ }^{\circ} \mathrm{C}$. Activation energies of the sintering process were determined from the dilatometric tests and the results showed that the decrease of calcareous content combined to the increase of flux content results in lower sintering temperatures, which can lead to lower energy costs.
\end{abstract}

Keywords: clay, ceramic tile, characterization, calcite, activation energy.

\section{INTRODUÇÃO}

A indústria de revestimentos cerâmicos tem tido um enorme crescimento nos últimos anos no Brasil, colocando o país em segundo lugar no ranking mundial como produtor e consumidor [1]. O Estado de Sergipe, com uma produção média de mais de dois milhões de metros quadrados por mês, vem buscando se enquadrar nesse processo, enfrentando atualmente o desafio de alcançar padrões aceitáveis de qualidade em um mercado altamente competitivo. As matérias-primas utilizadas na produção de revestimentos cerâmicos por via seca são predominantemente argilas que são constituídas pelos argilominerais ilita, caulinita, montmorilonita e ainda outros minerais que podem estar associados, como quartzo, calcita, hematita e feldspatos que se misturaram durante o processo de formação geológica $[2,3]$. As argilas devem apresentar plasticidade suficiente para conferir resistência mecânica na conformação por 
prensagem, de modo a garantir a integridade da peça no trajeto entre a prensa e o forno. O feldspato contido nas argilas são fontes dos óxidos de sódio e de potássio, atuando como fundentes em temperaturas acima de $1100{ }^{\circ} \mathrm{C}$ [4], o que facilita a formação de fase vítrea e reduz a porosidade. O quartzo é misturado com a argila durante formação geológica. Se está presente em menor proporção, auxilia na formação da fase vítrea, na desgaseificação da matéria orgânica e água. No entanto, grandes proporções de quartzo levam à drástica redução da resistência mecânica após a queima. O óxido de ferro está presente nas matérias-primas cerâmicas sob a forma de hematita ou goetita, conferindo a cor vermelha ao produto acabado. A calcita, que aparece na grande maioria das argilas utilizadas no processo de produção de revestimentos cerâmicos do tipo BIIb no estado de Sergipe [5], é um mineral que necessita de um cuidado especial em sua utilização devido à sua elevada perda ao fogo. Quando presente em proporção igual ou menor do que $3 \%$, esse mineral atua como fundente. No entanto, em proporções superiores, a calcita pode provocar aumento na porosidade final do produto. Além disso, o tamanho da partícula de calcita para processamento de cerâmicas deve ser inferior a $125 \mu \mathrm{m}$. Para tamanhos maiores, observase que o $\mathrm{CaO}$ resultante da dissociação dos carbonatos pode hidratar-se após a queima, promovendo variações na dimensão da peça [6]. Assim sendo, a utilização de argilas calcárias é um desafio, exigindo cuidado no processamento e controle na formulação e queima dos revestimentos.

Para garantir a sinterização correta do produto são necessárias a moagem e a prensagem adequadas da matériaprima, além de uma curva de queima eficiente, rápida e com o menor consumo energético possível. $\mathrm{O}$ estudo da cinética de sinterização pode ajudar a determinar as melhores condições de processamento, as quais correspondem à menor energia de ativação registrada em cada estágio. Esse conhecimento auxilia no desenvolvimento de curvas de queima adequadas [7]. O objetivo do presente trabalho foi avaliar a potencialidade de quatro argilas do estado de Sergipe a partir da caracterização física, químicomineralógica e microestrutural para aplicação na produção de revestimentos cerâmicos. Em cada caso, determinouse ainda a energia de ativação relacionada ao processo de queima, visando sua otimização.

\section{MATERIAIS E MÉTODOS}

Preparação das amostras: as argilas investigadas nesse trabalho são provenientes de quatro jazidas localizadas no estado de Sergipe, região nordeste do Brasil, conhecidas como: Ventinha (C1): coordenadas geográficas de latitude $10^{\circ} 05^{\prime} 10^{\prime \prime}$ Sul e longitude $37^{\circ} 08^{\prime} 43^{\prime \prime}$ Oeste; Poço Verde (C2): latitude $10^{\circ} 42^{\prime} 41^{\prime \prime}$ Sul e longitude 38 $11^{\prime} 6^{\prime \prime}$ " Oeste; Pau-de-leite (C3): latitude $10^{\circ} 42^{\prime} 41^{\prime \prime}$ Sul e longitude $38^{\circ} 11^{\prime} 06^{\prime \prime}$ Oeste; e Pinheiro (C4): latitude $10^{\circ} 48^{\prime} 12^{\prime \prime}$ Sul e longitude $37^{\circ} 10^{\prime} 11^{\prime \prime}$ Oeste. A Fig. 1 apresenta a posição de cada jazida em um mapa do Estado, onde cada região possui uma formação geológica peculiar. As amostras foram dispostas em bandejas e secas ao ar por $72 \mathrm{~h}$. Em seguida foram quarteadas, destorroadas e moídas em moinho de martelo com abertura de grelhas de $2 \mathrm{~mm}$.

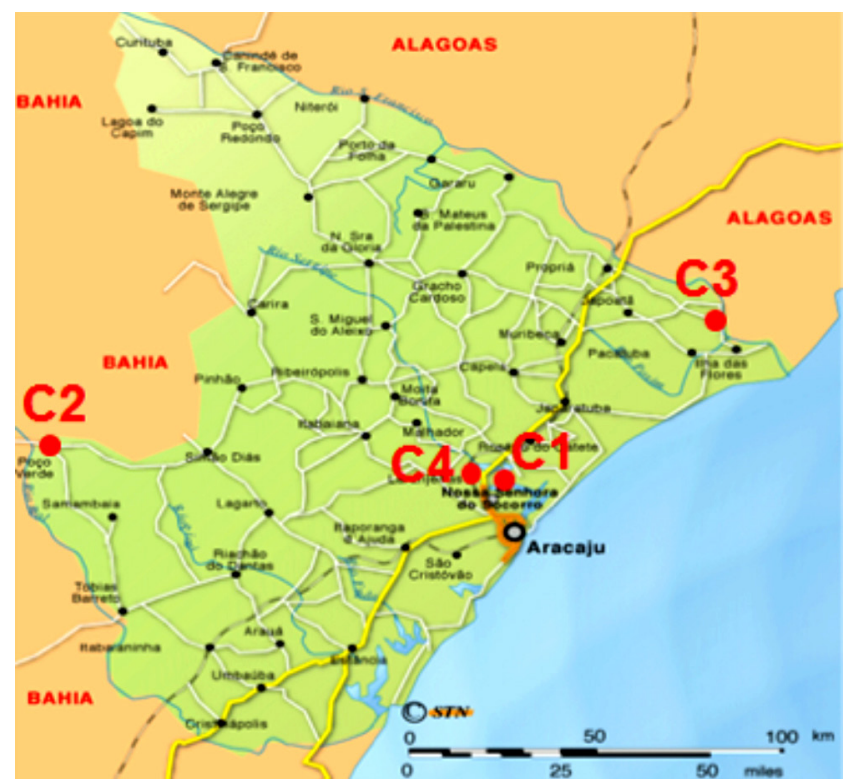

Figura 1: Localização dos depósitos de argila estudados nesse trabalho no estado de Sergipe, Brasil.

[Figure 1: Location of clay deposits studied in this work in Sergipe State, Brazil.]

Análises químicas: as composições químicas foram determinadas por fluorescência de raios X (FRX) em um equipamento Bruker S8 Tiger, no qual se determinou os percentuais dos óxidos constituintes pelo método semiquantitativo. Para essas medidas, as amostras com massa em torno de 10,0 g foram prensadas em forma cilíndrica com 40,0 mm de diâmetro e 4,0 $\mathrm{mm}$ de espessura. Durante as medidas, as amostras foram mantidas em vácuo de $10^{-6}$ bar. Uma mistura P-10 (90\% argônio e 10\% metano) foi utilizada no contador proporcional.

Composições mineralógicas: a técnica de difratometria de raios X (DRX) foi utilizada para a determinação das fases cristalinas. As amostras foram inicialmente secas em estufa a $100{ }^{\circ} \mathrm{C}$ por $24 \mathrm{~h}$, moídas e passadas por peneira de $150 \mu \mathrm{m}$. Os padrões de difração foram obtidos com um equipamento Rigaku D-MAX 100 utilizando radiação $\mathrm{K}_{\alpha, 1}$ do $\mathrm{Cu}(\lambda$ $=1,5418 \AA$ ). As medidas foram realizadas em modo de varredura contínua com velocidade de $1 \% \mathrm{~min}$, em intervalo de 5 a $65^{\circ}$. Amostras saturadas em etileno glicol por $1 \mathrm{~h}$ foram medidas em intervalo de 2 a $15^{\circ}$ para identificação da montmorilonita. As fases cristalinas dos minerais foram identificadas com o auxílio do programa de análise MATCH! (Phase Identification from Powder Diffraction), onde foram inseridos os arquivos cristalográficos do ICSD (Inorganic Crystal Structure Database).

Análises térmicas: medidas simultâneas de análise térmica diferencial (DTA) e termogravimétrica (TG) foram realizadas no equipamento SDT 2960 da TA Instruments para a investigação de processos endotérmicos 
e exotérmicos durante a etapa de aquecimento, como perda de água, desidroxilação da caulinita e dissociação da calcita. As medidas foram realizadas sob taxa de aquecimento $10{ }^{\circ} \mathrm{C} / \mathrm{min}$ no intervalo da temperatura ambiente até $1150{ }^{\circ} \mathrm{C}$, com fluxo de $100 \mathrm{~mL} / \mathrm{min}$ de ar sintético $\left(\mathrm{O}_{2} / \mathrm{N}_{2}-1 / 4\right)$. Um cadinho de platina vazio foi utilizado como referência nas medidas.

Análises dilatométricas: realizadas para verificar as alterações dimensionais de expansão e retração térmica de amostras, os ensaios dilatométricos foram feitos em um dilatômetro Netzsch DIL 402PC, sob fluxo de 130 $\mathrm{mL} / \mathrm{min}$ de ar sintético. Para esses ensaios, as amostras foram compactadas em forma cilíndrica com $12,0 \mathrm{~mm}$ de comprimento e $6,0 \mathrm{~mm}$ de diâmetro. Sob aquecimento a 10 ${ }^{\circ} \mathrm{C} / \mathrm{min}$, a temperatura foi monitorada desde a temperatura ambiente até $1150^{\circ} \mathrm{C}$. Através da análise cinética das curvas de dilatometria, foram determinadas as energias de ativação $\left(E_{a}\right)$ das argilas a partir da relação entre o logaritmo natural da retração linear com o inverso da temperatura, tendo como referência a região linear na fase final de sinterização, utilizando uma equação do tipo Arrhenius, no qual o coeficiente angular desta relação é o termo $-E_{a} / R$, sendo $R$ a constante dos gases ideais $[7,8]$. Supondo contração isotrópica dos corpos cerâmicos, a densidade ( $\rho$ ) foi calculada através da Equação A:

$$
\rho=\rho_{0} /\left(1-\Delta \mathrm{L} / \mathrm{L}_{0}\right)^{3}
$$

na qual $\mathrm{r}_{0}$ e $\mathrm{L}_{0}$ são a densidade e comprimento iniciais dos corpos cerâmicos a verde e $\Delta \mathrm{L}$ é a retração linear medida nos ensaios de dilatometria [8].

Preparação dos corpos de prova: foram conformados corpos de prova com dimensões de $120 \mathrm{~mm}$ x $56 \mathrm{~mm}$ x $6 \mathrm{~mm}$, utilizando-se uma prensa manual marca Somar com capacidade de 15 ton e pressão uniaxial de compactação de $28 \mathrm{MPa}$.

Ensaios de absorção de água: todos os corpos de prova foram sinterizados a $1130{ }^{\circ} \mathrm{C}$ com patamar de $3 \mathrm{~min}$ e ciclo de queima de $23 \mathrm{~min}$. Após a queima, as peças foram submetidas ao teste de absorção de água segundo a norma NBR 13818 [9], com imersão em água fervente por $2 \mathrm{~h}$ e pesagem antes e depois da imersão. Os resultados são expressos percentualmente (Equação $\mathrm{B}$ ), na qual $\mathrm{m}_{1}$ é a massa seca $\mathrm{e}_{2}$ a massa úmida, representadas com no mínimo uma casa decimal.

$$
\mathrm{AA}=\left[\left(\mathrm{m}_{2}-\mathrm{m}_{1}\right) / \mathrm{m}_{1}\right] .100
$$

Módulo de ruptura à flexão: a resistência mecânica dos corpos de prova sinterizados foi avaliada através da medida da variação do módulo de ruptura à flexão (MRF $)$ [9]. As medidas foram feitas em um flexômetro DT-090 da Servitech.

Distribuição de tamanho de partículas: para a determinação do tamanho de partículas das matérias-primas, utilizou-se o método de Fraunhofer [10] em um equipamento de medidas de difração de laser da Malvern, Mastersizer MS2000E, com agente dispersante.
Limites de Atterberg: os limites de liquidez (LL) e de plasticidade (LP) das matérias primas foram obtidos de acordo com a NBR 6459 e a NBR 7180, respectivamente $[11,12]$. O índice de plasticidade (IP) é o resultado da diferença aritmética entre os limites de liquidez e plasticidade (Equação C):

$$
\mathrm{IP}=\mathrm{LL}-\mathrm{LP}
$$

Microscopia eletrônica de varredura (MEV): esta técnica foi utilizada para identificação da forma e estrutura de poros nos corpos de prova sinterizados. As medidas foram realizadas em um microscópio eletrônico de varredura da Jeol, JSM-6510LV.

\section{RESULTADOS E DISCUSSÃO}

A Tabela I apresenta as composições químicas das argilas, determinadas por fluorescência de raios X. Os resultados mostram que todas as argilas são constituídas, principalmente, por $\mathrm{SiO}_{2}$ e $\mathrm{Al}_{2} \mathrm{O}_{3}$. Estes elementos são associados com estruturas de argilominerais, quartzo e feldspatos $[13,14]$. A maior quantidade de $\mathrm{SiO}_{2}$ foi determinada para a amostra C3. Esse componente é importante para a fabricação de revestimentos cerâmicos do tipo BIIb, uma vez que melhora a trabalhabilidade e favorece a compactação. No entanto, o $\mathrm{SiO}_{2}$ pode também provocar baixa resistência mecânica dos corpos cerâmicos sinterizados, além de reduzir a retração durante a queima [15]. A quantidade de $\mathrm{Fe}_{2} \mathrm{O}_{3}$ detectado nas amostras ficou entre $4,7 \%$ e 7,1\%. Esses valores são aceitáveis para uso em revestimentos cerâmicos do tipo BIIb, sendo este elemento responsável pela cor avermelhada das peças sinterizadas. Destaca-se o elevado teor de óxido de cálcio em C4 (12\%) e C1 (7\%), caracterizando essas argilas como calcárias [16]. $\mathrm{A}$ argila $\mathrm{C} 1$ foi previamente estudada em [13], que relata a formação de manchas em corpos cerâmicos produzidos com esse material, após sinterização a $1140{ }^{\circ} \mathrm{C}$. Este comportamento foi associado ao alto teor de $\mathrm{CaO}$, avaliado em $10 \%$. Este valor relatado é maior do que o determinado no presente trabalho (7\%). Considerando que a ref. [13] foi publicada em 2008, pode-se associar essa aparente discrepância à variação da concentração de $\mathrm{CaO}$ em diferentes extratos dessa jazida, que vem sendo continuamente lavrada. Analisando os teores de óxidos alcalinos, observa-se que a amostra $\mathrm{C} 2$ possui a maior concentração de $\mathrm{K}_{2} \mathrm{O}$, enquanto a concentração de $\mathrm{Na}_{2} \mathrm{O}$ é aproximadamente a mesma nas quatro amostras estudadas. Os compostos alcalinos e alcalino-terrosos possuem efeito fundente, o que facilita a formação de fase líquida e a retração linear durante a queima $[7,17]$. A Tabela II foi disposta de acordo com a quantidade crescente de $\mathrm{CaO}$ presente nas argilas. Nota-se que $\mathrm{C} 2$ e $\mathrm{C} 3$ possuem teor de $\mathrm{CaO}$ abaixo de $3 \%$. De acordo com [6], o $\mathrm{CaO}$ atua como fundente até o limite de $3 \%$ em massas de revestimento cerâmico. A porcentagem de óxidos de álcalis $\left(\mathrm{Na}_{2} \mathrm{O}\right.$ e $\left.\mathrm{K}_{2} \mathrm{O}\right)$, também apresentada na Tabela II, é outro fator preponderante para o processo de densificação, devido 
à grande tendência de formação de fase líquida durante a queima [6]. Considerando a soma dos percentuais de $\mathrm{CaO}$ e óxidos de álcalis nas amostras $\mathrm{C} 3$ e $\mathrm{C} 2$, pode-se concluir que $\mathrm{C} 2$ possui uma maior proporção de óxidos fundentes, sugerindo que essa amostra é a mais promissora. Por outro lado, as argilas com alto teor calcário, como é o caso de $\mathrm{C} 1$ e $\mathrm{C} 4$, tendem a apresentar maior porosidade e menor resistência mecânica após a queima [6]. Adicionalmente, essas duas matérias-primas possuem proporções de óxidos alcalinos inferiores aos observados para $\mathrm{C} 3$ e $\mathrm{C} 2$.

Tabela I - Composições químicas das argilas determinadas por FRX.

[Table I - Chemical compositions of clays determined by $X R F$.]

\begin{tabular}{ccccc}
\hline Óxidos $(\%)$ & $\mathrm{C} 1$ & $\mathrm{C} 2$ & $\mathrm{C} 3$ & $\mathrm{C} 4$ \\
\hline $\mathrm{SiO}_{2}$ & 50,2 & 52,1 & 63,0 & 45,3 \\
$\mathrm{Al}_{2} \mathrm{O}_{3}$ & 15,5 & 18,6 & 16,7 & 14,1 \\
$\mathrm{Fe}_{2} \mathrm{O}_{3}$ & 6,2 & 6,8 & 4,7 & 7,1 \\
$\mathrm{CaO}$ & 7,2 & 2,1 & 0,9 & 12,7 \\
$\mathrm{~K}_{2} \mathrm{O}$ & 3,2 & 4,7 & 3,8 & 3,2 \\
$\mathrm{Na}_{2} \mathrm{O}$ & 0,5 & 0,4 & 0,6 & 0,7 \\
$\mathrm{MgO}$ & 2,2 & 2,3 & 1,5 & 2,3 \\
$\mathrm{TiO}_{2}$ & 0,7 & 0,8 & 0,6 & 0,8 \\
Perda ao fogo & 14,3 & 12,1 & 8,2 & 13,8 \\
\hline
\end{tabular}

Tabela II - Percentuais de óxidos fundentes nas argilas estudadas, determinados por medidas de FRX.

[Table II - Percentage of fluxing oxides in clays determined via XRF measurements.]

\begin{tabular}{ccc}
\hline Argila & $\mathrm{CaO}(\%)$ & $\mathrm{Na}_{2} \mathrm{O}+\mathrm{K}_{2} \mathrm{O}(\%)$ \\
\hline $\mathrm{C} 3$ & 0,9 & 4,4 \\
$\mathrm{C} 2$ & 2,1 & 5,1 \\
$\mathrm{C} 1$ & 7,2 & 3,7 \\
$\mathrm{C} 4$ & 12,7 & 3,9 \\
\hline
\end{tabular}

Os padrões de difração das argilas são apresentados na Fig. 2A e se correlacionam positivamente com os resultados observados por fluorescência de raios X. As fases cristalinas foram identificadas de acordo com o banco de dados da ICSD (Inorganic Crystal Structure Database) e as principais fases identificadas foram quartzo, caulinita, muscovita, montmorilonita, calcita, feldspato e hematita. Os argilominerais caulinita e montmorilonita foram identificados em todas as argilas analisadas. De acordo com [18], esses argilominerais conferem a plasticidade necessária para garantir a conformação pelo processo de prensagem. Como pode ser observado na Fig. 2B, as amostras glicoladas apresentam deslocamento do pico de difração em torno de $2 \theta=5^{\circ}$, em relação às amostras naturais, devido à expansão dos seus parâmetros de rede. A distância interplanar basal do pico $\mathrm{d}_{001}$ da montmorilonita aumentou de $15,2 \AA$ para $17,1 \AA$ na amostra C1, de 15,4 $\AA$ para 17,2 $\AA$ em C2, de 15,1 $\AA$ para
$16,9 \AA$ em C3, e de 14,0 Å para 16,0 $\AA$ em C4. A porcentagem de cada fase cristalina presente nas amostras foi estimada a partir da intensidade relativa dos picos principais de cada fase [19], utilizando-se o programa de análise Match!. Os valores são apresentados na Tabela III e nota-se que as matérias-primas constituem-se principalmente de quartzo. A amostra C2 apresenta as maiores proporções dos minerais caulinita, muscovita e feldspato, o que é consistente com as concentrações de $\mathrm{Al}_{2} \mathrm{O}_{3}$ e $\mathrm{K}_{2} \mathrm{O}$ detectadas por FRX para essa amostra (Tabela I). O mineral feldspato desempenha um papel importante em materiais cerâmicos, atuando como agente de fluxo para reduzir as temperaturas de fusão das argilas e conferir alta densificação após queima $[18,20]$. A porcentagem de calcita variou consideravelmente entre as amostras estudadas, com extremos de 1,1\% em C3 e 13,7\% em C4.

A Fig. 3 apresenta as curvas de distribuição de tamanho de partículas para as quatro matérias-primas estudadas. Observa-se o perfil aproximadamente Gaussiano em todas
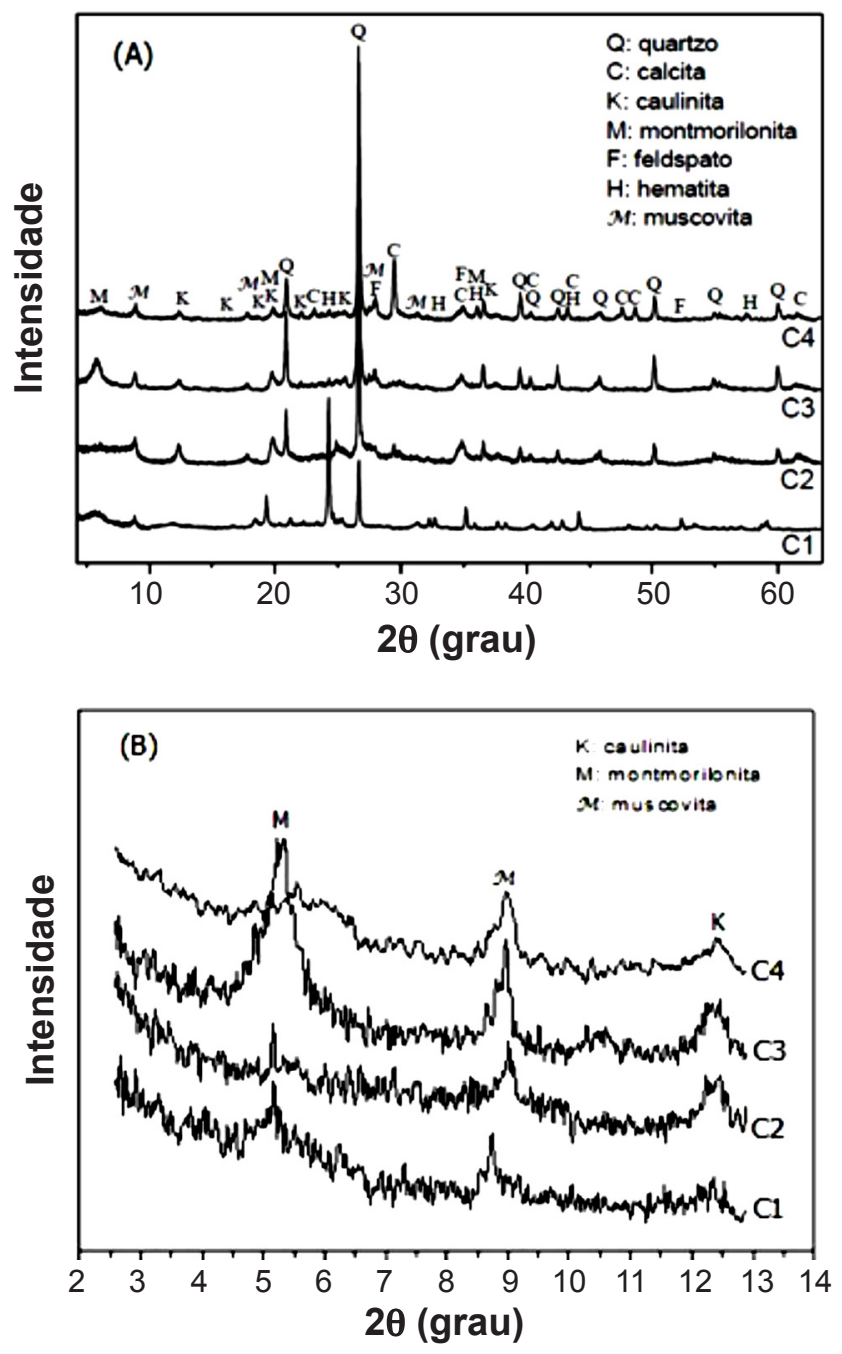

Figura 2: Difratogramas de raios $\mathrm{X}$ das argilas e seus respectivos minerais (A) e das argilas glicoladas (B).

[Figure 2: XRD patterns of clays and their minerals (A), and of clays treated with ethylene glycol (B).] 
Tabela III - Composições mineralógicas das argilas determinadas por DRX.

[Table III - Mineralogical compositions of clays determined by XRD.]

\begin{tabular}{ccccc}
\hline Minerais (\%) & C1 & C2 & C3 & C4 \\
\hline Quartzo & 55,7 & 51,8 & 65,1 & 57,1 \\
Caulinita & 6,3 & 10,7 & 7,4 & 5,5 \\
Muscovita & 11,8 & 14,0 & 11,2 & 12,1 \\
Montmorilonita & 5,6 & 4,9 & 4,6 & 6,7 \\
Calcita & 8,6 & 2,8 & 1,1 & 13,7 \\
Feldspato & 6,3 & 9,9 & 6,2 & 3,2 \\
Hematita & 5,7 & 5,9 & 4,4 & 1,7 \\
\hline
\end{tabular}

as curvas, o que confirma a distribuição homogênea das partículas [21]. A proporção de partículas com diâmetro menor que $2 \mu \mathrm{m}$ (fração argilosa) é fator determinante para a plasticidade do material. Matérias-primas utilizadas para a produção de revestimento necessitam de plasticidade suficiente para garantir a conformação do produto por prensagem [22]. Na Tabela IV, que apresenta a distribuição de tamanho de partículas e seu diâmetro médio, observa-se que a amostra C2 apresenta a maior fração argilosa entre os materiais estudados no presente trabalho. Adicionalmente,

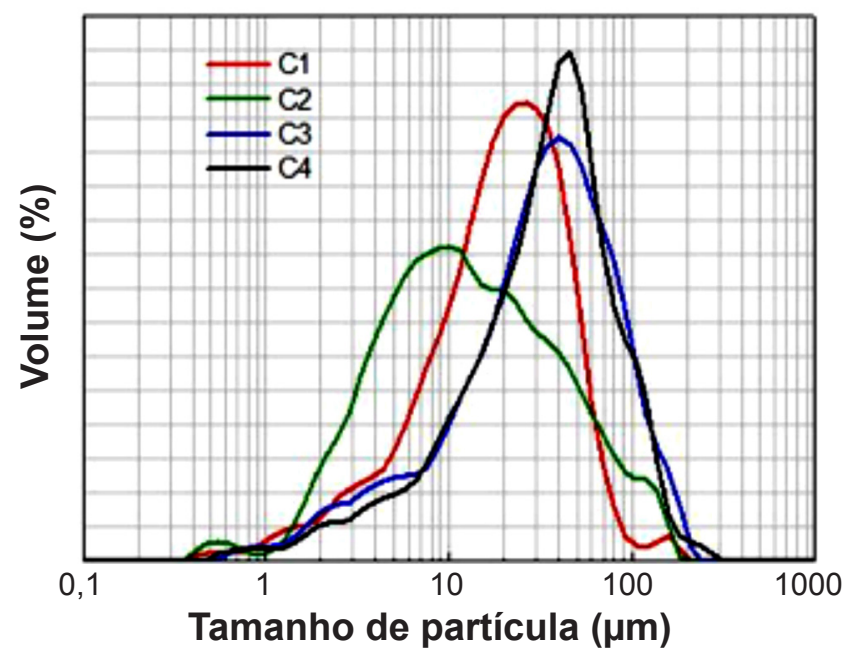

Figura 3: Curvas de distribuição de tamanho de partículas das argilas determinadas por difração de laser.

[Figure 3: Particle size distribution curves of clays determined by laser diffraction.] essa amostra apresentou também o menor diâmetro médio e ausência de partículas acima de $200 \mu \mathrm{m}$. Por outro lado, os maiores valores de tamanho de partícula se situam entre 20 e $200 \mu \mathrm{m}$, com a menor fração argilosa e o maior diâmetro médio determinados para a amostra $\mathrm{C} 4$. Os limites de Atterberg das argilas são também apresentados na Tabela IV. Materiais que apresentam IP entre 1 e 7\% são considerados fracamente plásticos, de 7 a $15 \%$ são medianamente plásticos e acima de $15 \%$ altamente plásticos [23]. De acordo com essa classificação, os valores de índices de plasticidade (IP) determinados para C1, C2 e C3 indicam que essas argilas são altamente plásticas (IP $>15 \%$ ). Por outro lado, C4 é moderadamente plástica, com IP $=15 \%$. O valor mínimo ideal para a produção cerâmica é de $15 \%$ [24], indicando que as quatro argilas estudadas podem ser utilizadas para este fim. A elevada plasticidade está associada com produtos com uma maior resistência mecânica após prensagem [25]. Observa-se uma relação direta entre o IP e a fração argilosa das amostras, mais uma vez apontando para o melhor desempenho da matéria-prima C2. Os parâmetros LL e LP dependem, geralmente, dos argilominerais presentes na amostra, os quais podem expandir ou se retrair em função da umidade. No presente trabalho, o maior valor de LL foi determinado para a amostra $\mathrm{C} 2$, enquanto a amostra $\mathrm{C} 3$ apresentou o maior valor de LP.

As curvas de TG/DTG-DTA são apresentadas na Fig. 4 e podem ser interpretadas à luz das transformações químicas e mineralógicas que ocorrem em cada amostra [26]. Os eventos observados abaixo de $110{ }^{\circ} \mathrm{C}$ em todas as curvas são devidos à perda de água adsorvida [27]. Em particular, para materiais contendo montmorilonita, a perda de massa observada durante o aquecimento entre 50 a $200{ }^{\circ} \mathrm{C}$ corresponde à liberação de moléculas de água a partir da zona de hidratação hidrofóbica situada entre as folhas de silicato. Todas as curvas de DTA (Fig. 4B) apresentam um pico endotérmico em torno de $480{ }^{\circ} \mathrm{C}$, acompanhado por perda de massa registrada na Fig. 4A. Este evento térmico corresponde à desidroxilação da caulinita, que consiste na remoção da água estrutural, seguida pela formação de metacaulinita [28]. Uma perda de massa considerável ocorre em torno de $680^{\circ} \mathrm{C}$ e pode estar associada a dois eventos térmicos que ocorrem em temperaturas próximas. Um deles é a desidroxilação da montmorilonita [28] e o outro é a decomposição térmica do $\mathrm{CaCO}_{3}$, resultando em $\mathrm{CaO}$ e gás $\mathrm{CO}_{2}[7,8,29-32]$, com efeito deletério sobre a densificação [33]. As maiores perdas de massa a estas temperaturas são observadas para $\mathrm{C} 4$ e $\mathrm{C} 1$,

Tabela IV - Frações em faixas de tamanho, diâmetro médio de partículas e limites de Atterberg das argilas. [Table IV - Fractions in size ranges, average particle diameter, and Atterberg limits of clays.]

\begin{tabular}{ccccccccc}
\hline \multirow{2}{*}{ Amostra } & \multicolumn{3}{c}{ Fração (\% em massa) na faixa de tamanhos de partículas } & \multicolumn{3}{c}{ Limites de Atterberg (\%) } \\
& $<2 \mu \mathrm{m}$ & $2-20 \mu \mathrm{m}$ & $20-200 \mu \mathrm{m}$ & $>200 \mu \mathrm{m}$ & $\mathrm{d}_{\mathrm{m}}(\mu \mathrm{m})$ & LL & LP & IP \\
\hline $\mathrm{C} 1$ & 4,25 & 46,6 & 48,7 & 1,5 & 25,0 & 52 & 22 & 30 \\
$\mathrm{C} 2$ & 6,32 & 58,9 & 36,3 & - & 6,5 & 59 & 25 & 34 \\
$\mathrm{C} 3$ & 3,56 & 26,4 & 67,6 & 2,9 & 26,5 & 49 & 30 & 19 \\
$\mathrm{C} 4$ & 2,87 & 24,5 & 71,5 & 1,9 & 30,0 & 39 & 24 & 15 \\
\hline
\end{tabular}



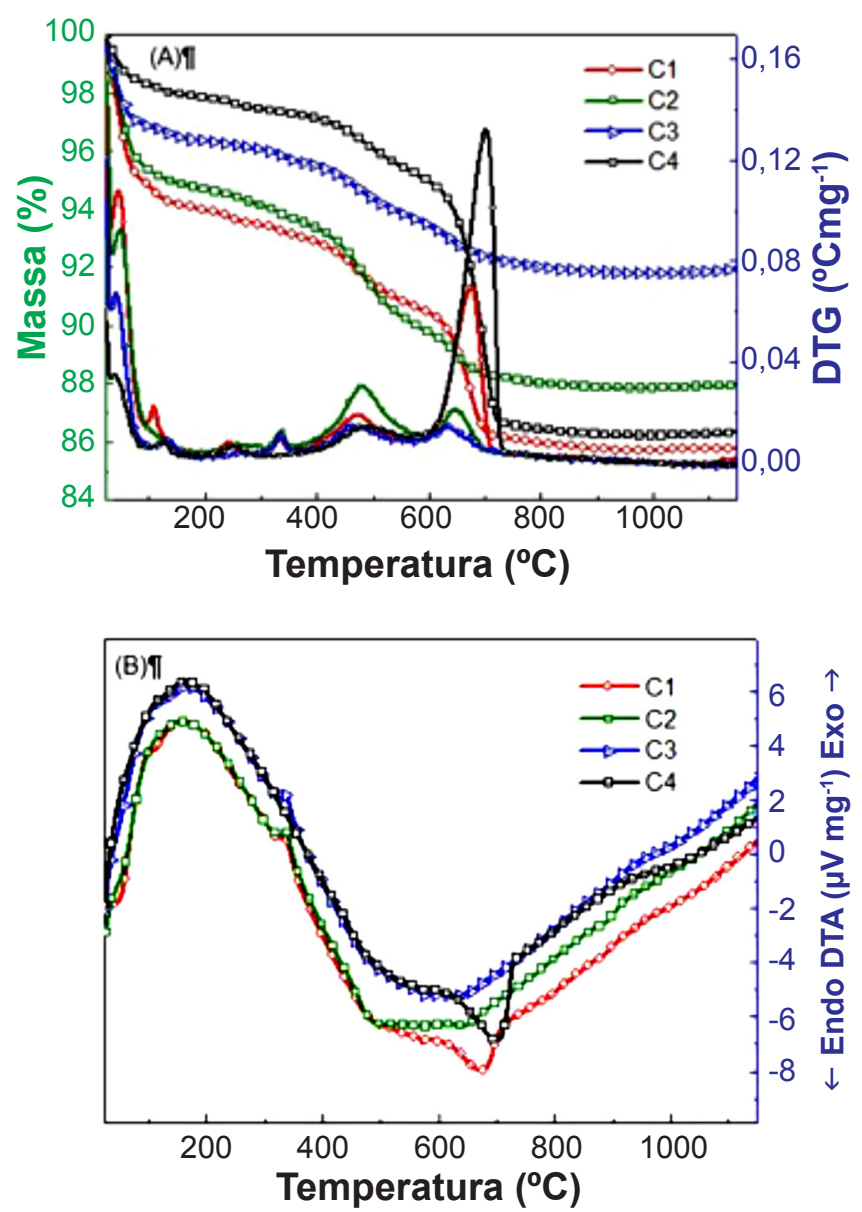

Figura 4: Resultados de análise térmica das amostras: (A) curva termogravimétrica e sua primeira derivada (TG/DTG), (B) análise térmica diferencial (DTA).

[Figure 4: Results of thermal analysis of the samples: (A) thermogravimetric curve and its first derivative (TGA/DTG), (B) differential thermal analysis (DTA).]

o que é consistente com o fato de que essas duas amostras apresentaram os maiores teores de calcita e montmorilonita (Tabela III). A decomposição dos carbonatos, observada entre 650 e $700{ }^{\circ} \mathrm{C}$ no presente trabalho, está abaixo do valor de $800{ }^{\circ} \mathrm{C}$ relatado em [21]. O fato está provavelmente associado ao grau de cristalinidade do $\mathrm{CaCO}_{3}$, como descrito por outros pesquisadores [29, 30]. A Tabela $\mathrm{V}$ apresenta as perdas de massa determinadas a partir da área sob os picos de DTG a 480 e $680^{\circ} \mathrm{C}$, bem como a perda de massa total de cada argila. Os valores obtidos são consistentes com as fases mineralógicas de cada amostra (Tabela III). A amostra C2 apresentou a maior concentração de caulinita $(10,7 \%)$ e a maior perda de massa $(4,0 \%)$ a $480{ }^{\circ} \mathrm{C}$. Por outro lado, a amostra $\mathrm{C} 4$ apresentou as concentrações mais elevadas de calcita $(13,7 \%)$ e apresentou a maior perda de massa $(4,8 \%)$ a $680{ }^{\circ} \mathrm{C}$. A menor e a maior perda de massa foram observadas para as amostras $\mathrm{C} 3$ e $\mathrm{C} 1$, respectivamente, provavelmente devido à quantidade de argilominerais e carbonato de cálcio. Acima de $1150^{\circ} \mathrm{C}$ ocorre o início da dissolução do quartzo, fato que deve ser evitado na produção de revestimentos cerâmicos, devido à demasiada retração linear e diminuição
Tabela V - Perdas de massa (\%) determinadas por medidas de termogravimetria das argilas.

[Table V-Mass loss (\%) determined from thermogravimetric measurements of the clays.]

\begin{tabular}{ccccc}
\hline Temperatura $\left({ }^{\circ} \mathrm{C}\right)$ & $\mathrm{C} 1$ & $\mathrm{C} 2$ & $\mathrm{C} 3$ & $\mathrm{C} 4$ \\
\hline 480 & 2,9 & 4,0 & 1,8 & 1,5 \\
680 & 4,1 & 1,7 & 0,9 & 4,8 \\
1150 & 14,3 & 12,1 & 7,3 & 13,8 \\
\hline
\end{tabular}

da resistência mecânica do produto final [17].

As curvas dilatométricas das argilas são mostradas na Fig. 5. Observou-se nessas curvas uma ligeira expansão até aproximadamente $8500^{\circ} \mathrm{C}$, sendo que a $573^{\circ} \mathrm{C}$ a expansão foi mais acentuada devido à transformação do quartzo $\alpha$ para $\beta$ em todas as amostras [34], exceto em C2 que apresenta menor porcentagem de quartzo livre (Tabela III). A partir de $573{ }^{\circ} \mathrm{C}$, houve uma redução gradual da taxa de expansão, ocorrendo o início da sinterização, seguida de uma retração exponencial [34]. A retração linear apresentada por C2 é notadamente maior do que a observada para as demais

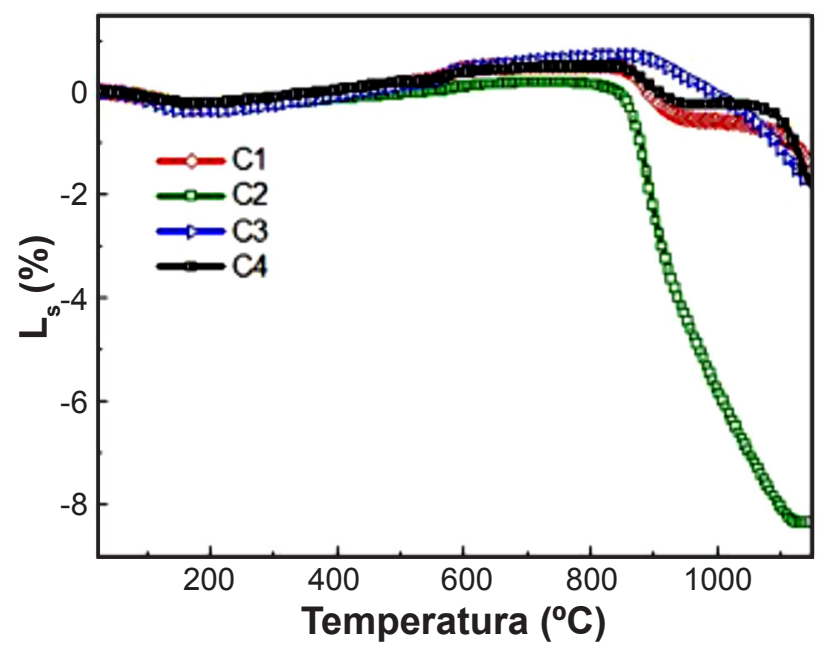

Figura 5: Curvas dilatométricas das argilas à taxa de aquecimento de $10^{\circ} \mathrm{C} / \mathrm{min}$.

[Figure 5: Dilatometric curves of clays at a heating rate of $10^{\circ} \mathrm{C} / \mathrm{min}$.]

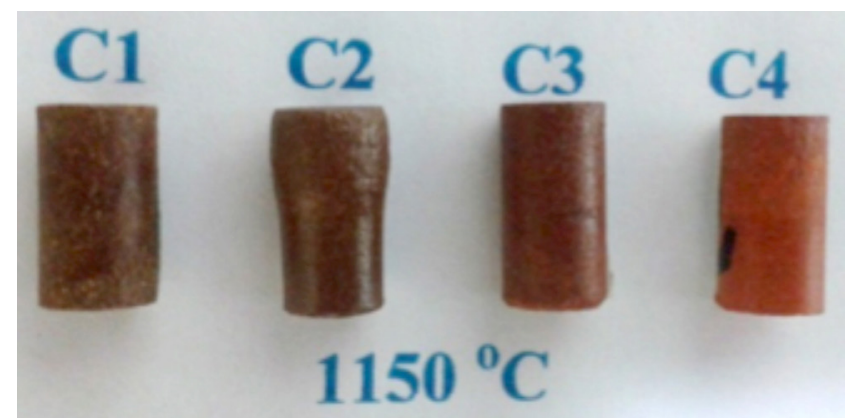

Figura 6: Corpos sinterizados a $1150{ }^{\circ} \mathrm{C}$, onde se observa a deformação da amostra $\mathrm{C} 2$.

[Figure 6: Ceramic bodies sintered at $1150{ }^{\circ} \mathrm{C}$, showing the deformation of sample C2.] 
amostras. Este comportamento deve-se à maior quantidade de fundentes, ou seja, carbonato de cálcio (até o limite de 3\%) + óxidos alcalinos (Tabela II). Além disso, a distribuição do tamanho de partículas e o índice de plasticidade favorecem a compactação e, consequentemente, a densificação. Apesar das vantagens em relação às demais amostras, a argila $\mathrm{C} 2$ sofreu deformação durante a queima até $1150{ }^{\circ} \mathrm{C}$, como pode ser observado na Fig. 6. Este efeito, conhecido como deformação piroplástica, pode se dever à grande proporção de fundentes na amostra. Uma das formas de controle da deformação durante a queima é o ajuste da temperatura de queima, para que a placa continue dentro das normas exigidas [35].

A densidade do corpo cerâmico sinterizado possui correlação direta com a absorção de água e o módulo de resistência à flexão, pois quanto maior for a densificação, menor será a absorção de água e porosidade [3, 9]. Na Fig. 7 temos a evolução da densidade como uma função da temperatura de queima, calculada a partir da relação com a retração linear nas medidas de dilatometria (Equação A). Vale destacar o aumento da densidade na amostra $\mathrm{C} 2 \mathrm{em}$ relação às demais, atingindo o valor máximo de $2,55 \mathrm{~g} / \mathrm{cm}^{3}$ a $1120^{\circ} \mathrm{C}$. Observa-se a partir da Tabela VI os resultados dos ensaios tecnológicos de absorção de água (AA), módulo de ruptura à flexão $(\mathrm{MRFq})$ e retração linear $\left(\mathrm{R}_{\mathrm{L}}\right)$ dos corpos queimados a $1130{ }^{\circ} \mathrm{C}$. Para que uma argila atenda às especificações da norma para a produção de revestimento cerâmico do tipo BIIb, a absorção de água deve ser de 6 a $10 \%$ e a resistência mecânica à flexão maior do que $18 \mathrm{MPa}$ [9]. A amostra C2 foi a única a atender essas especificações entre as argilas estudadas (Fig. 8). Isto se deve à maior porcentagem de álcalis, sódio + potássio, combinada com a porcentagem de $\mathrm{CaO}$, o que conduz à formação de fase líquida acima de $1000{ }^{\circ} \mathrm{C}$. Assim, o líquido formado escoa,

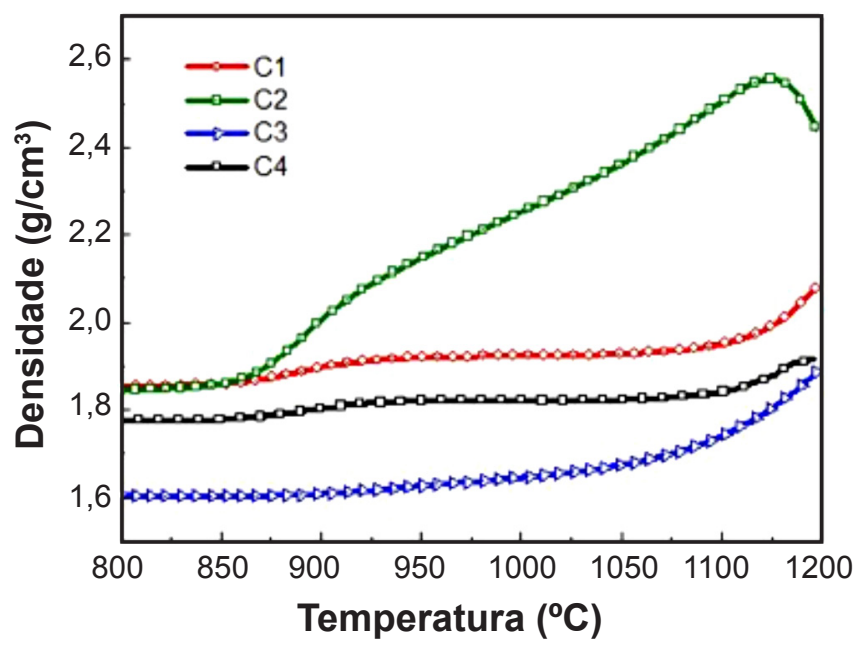

Figura 7: Densidade como uma função da temperatura de queima, calculada a partir da relação com a retração linear nas medidas de dilatometria.

[Figure 7: Density as a function of firing temperature, calculated from the relationship with the linear shrinkage in dilatometry measurements.]
Tabela VI - Valores de absorção de água, módulo de ruptura à flexão e retração total das argilas.

[Table VI - Values of water absorption, flexural modulus of rupture, and full retraction of clays.]

\begin{tabular}{cccc}
\hline Amostra & $\begin{array}{c}\text { Absorção } \\
\text { de água } \\
(\%)\end{array}$ & $\begin{array}{c}\text { Módulo de } \\
\text { ruptura à } \\
\text { flexão (MPa) }\end{array}$ & $\begin{array}{c}\text { Retração total } \\
1130{ }^{\circ} \mathrm{C}(\%)\end{array}$ \\
\hline $\mathrm{C} 1$ & 18,0 & 43,8 & 1,2 \\
$\mathrm{C} 2$ & 9,2 & 48,1 & 8,3 \\
$\mathrm{C} 3$ & 14,5 & 44,1 & 1,6 \\
$\mathrm{C} 4$ & 15,1 & 44,2 & 1,5 \\
\hline
\end{tabular}

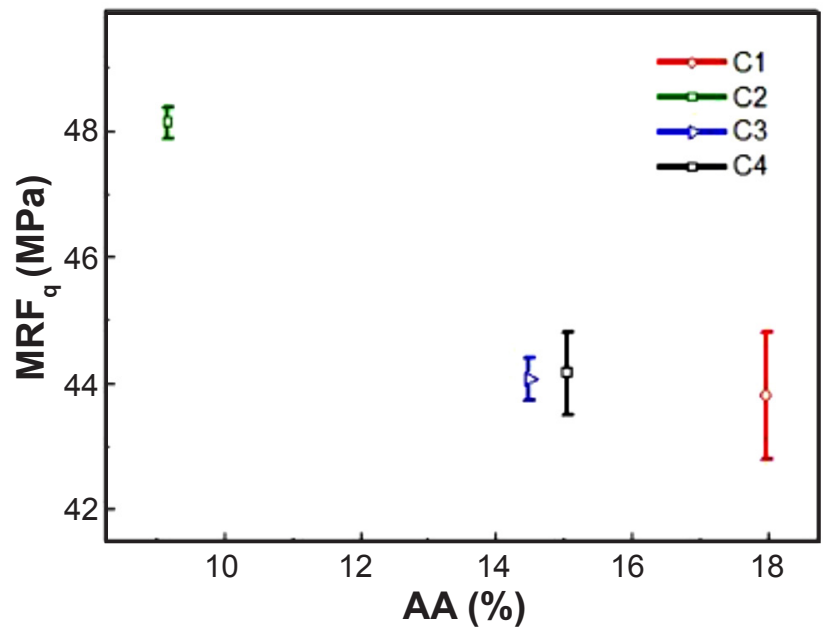

Figura 8: Variação do módulo de ruptura à flexão (MRFq) com a absorção de água (AA) das argilas.

[Figure 8: Variation of the modulus of rupture in bending (MRFq) with the water absorption (AA) of clays.]

preenchendo os espaços vazios e, por força da capilaridade, promove a aproximação das partículas, aumentando o adensamento do corpo cerâmico [36]. Nas condições de processamento adotadas, placas cerâmicas produzidas a partir das matérias-primas $\mathrm{C} 1, \mathrm{C} 3$ e $\mathrm{C} 4$ classificam-se no grupo BIII, pois apresentaram absorção de água superior a $10 \%$.

Nas imagens de microscopia eletrônica de varredura (Fig. 9) realizadas da superfície de fratura das argilas sinterizadas a $1150{ }^{\circ} \mathrm{C}$, nota-se em $\mathrm{C} 1, \mathrm{C} 3$ e $\mathrm{C} 4$ que a fase líquida não estava completamente distribuída pela estrutura. A argila C2 mostra que a configuração da estrutura é de maior densificação. A porosidade aberta vai sendo eliminada e a superfície de fratura vai mudando de aspecto, tornandose mais densa e regular [37]. Nessa faixa de temperaturas ocorre maior formação de fase líquida, a qual se distribui melhor pela estrutura, promovendo maior redução do volume de poros, resultando também em uma estrutura mais resistente. Essa microestrutura densa é responsável pelas propriedades físicas e mecânicas obtidas para C2 (Tabela VI e Fig. 8).

No presente trabalho foi dada uma atenção especial para o comportamento da retração durante o estágio final 


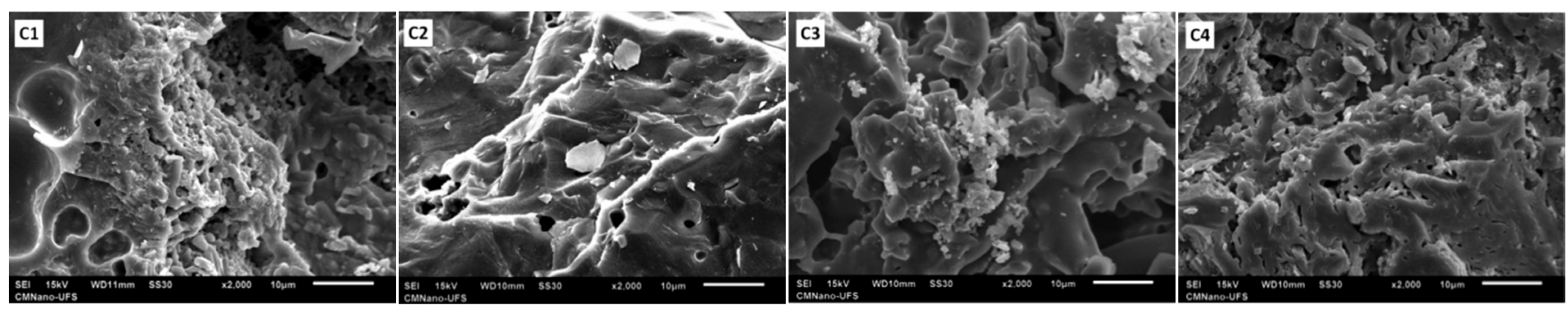

Figura 9: Micrografias obtidas por microscopia eletrônica de varredura da superfície de fratura das argilas sinterizadas a $1150{ }^{\circ} \mathrm{C}$. [Figure 9: SEM micrographs of the fracture surface of clays sintered at $1150^{\circ} \mathrm{C}$.]
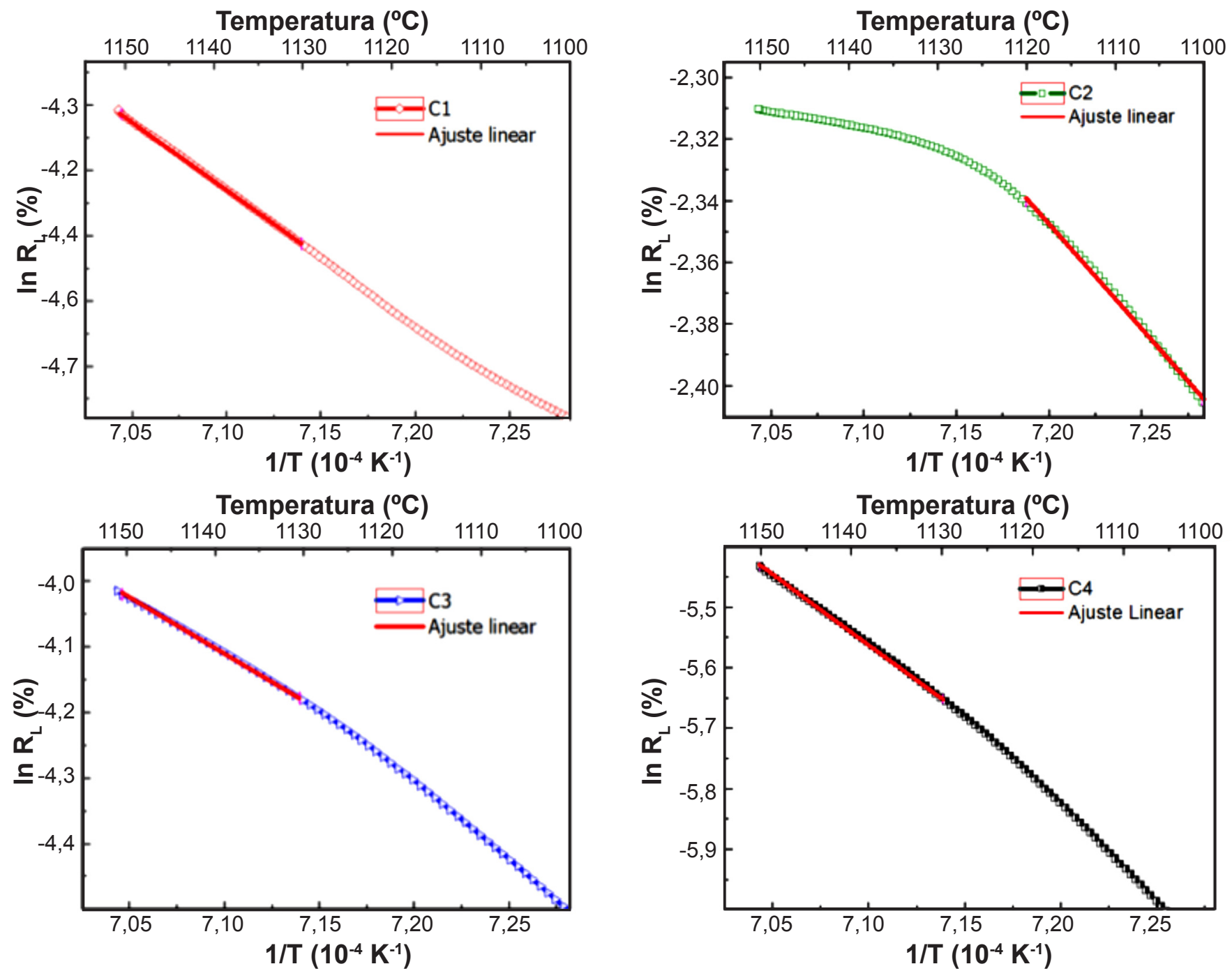

Figura 10: Gráficos de Arrhenius e os ajustes lineares encontrados para cada argila na região linear das curvas de dilatometria na etapa final de sinterização.

[Figure 10: Arrhenius plot and linear fit found for each clay in linear region of the dilatometric curves in final sintering stage.]

Tabela VII - Energias de ativação de sinterização das argilas nos intervalos de temperatura indicados.

[Table VII - Activation energies of sintering for clays in the indicated temperature ranges.]

\begin{tabular}{cccc}
\hline Amostra & Intervalo de temperatura $\left({ }^{\circ} \mathrm{C}\right)$ & Energia de ativação $(\mathrm{kJ} / \mathrm{mol})$ & $\mathrm{R}^{2}$ \\
\hline C1 & 1130 a 1150 & 174 & 0,99988 \\
C2 & 1100 a 1120 & 57 & 0,99714 \\
C3 & 1130 a 1150 & 197 & 0,99943 \\
C4 & 1130 a 1150 & 188 & 0,99947 \\
\hline
\end{tabular}


de sinterização na região linear das curvas de dilatometria, que podem ajudar a estabelecer uma curva de queima apropriada para fins industriais. A Fig. 10 mostra os gráficos do logaritmo natural da retração linear em função do inverso da temperatura absoluta para cada argila. A região linear na fase final de sinterização desses gráficos pode ser ajustada de acordo com a equação de Arrhenius, dada por $[7,8]$ :

$$
\ln \mathrm{R}_{\mathrm{L}}=\left(-\mathrm{E}_{\mathrm{a}} / \mathrm{RT}\right)+1 / \mathrm{T}
$$

Pela análise dessa equação, percebe-se que o gráfico de $\ln \mathrm{R}_{\mathrm{L}}$ em função do inverso da temperatura absoluta é linear, com coeficiente angular igual a $-E_{\alpha} / R$, sendo $R$ a constante dos gases ideais e $E_{a}$ a energia de ativação para o processo. Na Fig. 10 são apresentados os dados experimentais de retração linear e os ajustes realizados na região em que a Equação D é atendida. Os resultados das energias de ativação obtidos para cada ajuste nos respectivos intervalos de temperatura são mostrados na Tabela VII, na qual se observa o menor valor para C2. A energia de ativação representa a altura da barreira de potencial para que ocorram processos de difusão durante a queima. Baixos valores de energia de ativação significam maior facilidade para transporte de massa durante a queima, de modo que esse transporte ocorre a temperaturas mais baixas e de forma mais rápida. Esse resultado demonstra que o uso da matéria-prima $\mathrm{C} 2$ resulta em uma sinterização mais rápida e com menor custo energético, o que é um resultado bastante promissor para a redução de custos na produção de revestimentos cerâmicos. Para as demais amostras, os resultados das energias de ativação estão compatíveis com a ref. [7], que encontrou valores médios de $178 \mathrm{~kJ} / \mathrm{mol}$ para materiais utilizados na produção de revestimentos cerâmicos do tipo BIIa. A boa concordância entre os resultados e a referência valida a metodologia utilizada no presente trabalho.

\section{CONCLUSÕES}

Foram estudadas quatro diferentes argilas utilizadas no segmento de produção de revestimento cerâmico do tipo BIIb no estado de Sergipe. Caracterizações físicas, químicomineralógicas e microestruturais, bem como a determinação das energias de ativação no processo de sinterização, foram realizadas visando à otimização da queima. Todas as argilas apresentaram em sua composição o mineral calcita que, a depender da porcentagem, influi diretamente nas propriedades do produto. Os resultados mostraram que a decomposição do carbonato de cálcio retarda o processo de sinterização e a evolução do $\mathrm{CO}_{2}$ favorece a porosidade aberta, desempenhando um papel importante no desenvolvimento das propriedades tecnológicas das peças. Nas argilas C1, C3 e C4, há um maior volume de poros, já em C2 a configuração da estrutura é mais densa, evidenciando a formação de fase líquida, promovendo a redução do volume de poros. Essa microestrutura densa é responsável pelas propriedades físicas e mecânicas obtidas para $\mathrm{C} 2$, demonstrando a importância da combinação das quantidades de óxidos de álcalis, do teor de calcita e do tamanho das partículas. O índice de plasticidade obteve valores satisfatórios para conformação por prensagem de revestimento do tipo $\mathrm{BIIb}$ em todas as amostras. O menor valor de energia de ativação em $\mathrm{C} 2$ indica que nessa argila as reações ocorrem mais rapidamente. Isto demonstra que o uso dessa matéria-prima pode resultar em curvas de queima mais rápidas e eficientes. É previsível que o presente trabalho vai ajudar a melhorar o conhecimento sobre essas argilas, bem como contribuir para a exploração dos depósitos e a otimização na fabricação de revestimentos cerâmicos no Estado.

\section{AGRADECIMENTOS}

Os autores agradecem às agências de financiamento FAPITEC/SE, CNPq, CAPES, FINEP, ao CMNano (Centro Multiusuário de Nanotecnologia da UFS) e à indústria de revestimentos cerâmicos Cerâmica Sergipe S.A.

\section{REFERÊNCIAS}

[1] A.O. Boschi, "Desempenho da Indústria Brasileira de Revestimentos Cerâmicos entre 2009 e 2013", Cerâmica Industrial 19, 5 (2014) 7.

[2] J.A. Cornell, Experiments and mixtures, John Wiley Sons, New York (1997) 23.

[3] Associação Nacional de Fabricantes de Cerâmica, ANFACER. Disponível em http://www.anfacer.org.br, acesso em 23/07/2015.

[4] R.J.S. Castro, "Estudo do efeito do feldspato e resíduo de caulim na produção de revestimento cerâmico", Cerâm. Ind. 20, 1 (2015) 30.

[5] J.R. Goes, T.F. Azevedo, T.X.C. Dutra, V.B. Santos, J.B. Severo Junior, L.S. Barreto, "Avaliação da potencialidade de argilas da formação geológica Calumbi e Riachuelo em Sergipe para aplicação em revestimento cerâmico", Cerâmica 60 (2014) 211.

[6] E.J. Enrique, "Decomposición de carbonatos durante la coccion de piezas de revestimiento cerâmico vidreado", Relacion com la aparicion de pinchados, Qualicer (1998).

[7] M. Cargnin, S.M.A.G. Souza, A.A.U. Souza, A.J. Noni, "Determinação de parâmetros cinéticos da sinterização de revestimentos cerâmicos de monoqueima do tipo BIIa", Cerâmica 57 (2011) 461.

[8] M.N. Rahaman, Ceramic Processing and Sintering, 2nd Ed., Missouri, Taylor \& Francis (2003) 782.

[9] Associação Brasileira de Normas Técnicas, NBR 13818: Placas cerâmicas para revestimento, Especificação e métodos de ensaio, Rio de Janeiro (1997).

[10] P. Kippax, Measuring particle size using modern laser diffraction techniques. Paint \& Coatings Industry, (2005). Disponível em: http://www.malvern.com/malvern/kbase. nsf/allbyno/KB000930/\$file/MRK696-01.pdf.

[11] Associação Brasileira de Normas Técnicas, NBR 7180: 
Determinação do limite de liquidez de solos, Rio de Janeiro (1984).

[12] Associação Brasileira de Normas Técnicas, NBR 6459: Determinação do limite de plasticidade de solos, Rio de Janeiro (1984).

[13] A.C. Alcântara, M.S. Beltrão, H.A. Oliveira, I.F. Gimenez, L.S. Barreto, "Characterization of ceramic tiles prepared from two clays from Sergipe-Brazil", Appl. Clay Sci. 39 (2008) 160.

[14] M. Dondi, G. Guarini, P. Ligas, M. Palomba, M. Raimondo, I. Uras, "Chemical, mineralogical and ceramic properties of kaolinitic materials from the Tresnuraghes mining district (Western Sardinia, Italy)", Appl. Clay Sci. 18, (2001) 145.

[15] M. Seynou, Y. Millogo, R. Ouedraogo, K. Traore, J. Tirlocq, "Firing transformations and properties of tiles from a clay from Burkina Faso", Appl. Clay Sci. 51 (2011) 499.

[16] F. Gonzalez, V. Romero, G. Garcia, M. Gonzalez, "Firing transformations of mixtures of clays containing illite, kaolinite and calcium carbonate used by ornamental tile industries", Appl. Clay Scie. 5 (1990) 361.

[17] S.Y.R. López, J.S. Rodriguez, S.S. Sueyoshi, "Determination of the activation energy for densification of porcelain stoneware", J. Ceram. Proc. Res. 12 (2011) 228.

[18] H. Celik, "Technological characterization and industrial application of two Turkish clays for the ceramic industry", Appl. Clay Sci. 50 (2010) 245.

[19] Z.S. Macedo, C.R. Ferrari, A.C. Hernandes, "Selfpropagation high-temperature synthesis of bismuth titanate", Powder Technol. 139 (2004) 175.

[20] S.K. Das, K. Dana, "Differences in densification behaviour of $\mathrm{K}$ - and Na-feldspar containing porcelain bodies", Thermochim. Acta, 406, 1 (2003) 199.

[21] F.H. Zaied, R. Abidi, N. Slim-Shimi, A.K. Somarin, "Potentiality of clay raw materials from Gram area (Northern Tunisia) in the ceramic industry", Appl. Clay Sci. 112-113 (2015) 1.

[22] P.S. Santos, Ciência e tecnologia de argilas, $3^{\text {a }}$ Ed., Edgard Blucher Ltda, S. Paulo (1997) 23.

[23] S.C. Maestrelli, C.D. Roveri, A.G.P. Nunes, L.M. Faustino, G.F. Aielo, L.P.A. Pinto, C. Manochio, T.M.L. Cal, F.F. Ribeiro, N.A. Mariano, "Estudo de caracterização de argilas não plásticas da região de Poços de Caldas, MG", Cerâmica 59 (2013) 242.

[24] S. Mahmoudi, E. Srasra, F. Zargouni, "The use of Tunisian Barremian clay in the traditional ceramic industry: optimization of ceramic properties", Appl. Clay Sci. 42 (2008) 125.

[25] J.M.M. Maroto, J.A.A. Azcárate, “An accurate, quick and simple method to determine the plastic limit and consistency changes in all types of clay and soil: The thread bending test", Appl. Clay Sci. 114 (2015) 497.

[26] L. Mota, R. Toledo, A.L. Machado, J. Holanda, H. Vargas, R. Faria, "Thermal characterization of red clay from the Northern Region of Rio de Janeiro State, Brazil using an open photoacoustic cell, in relation to structural changes on firing", Appl. Clay Sci. 42 (2008) 168.

[27] P.B. Arab, O.J. Pejon, "Identification of clay minerals in mixtures subjected to differential thermal and thermogravimetry analyses and methylene blue adsorption tests", Appl. Clay Sci. 114 (2015) 133.

[28] P. Ptacek, D. Kubatova, J. Havlika, J. Brandstetr, F. Soukal, T. Opravil, "The non-isothermal kinetic analysis of the thermal decomposition of kaolinite by thermogravimetric analysis", Powder Technol. 204 (2010) 222.

[29] P. Cardiano, S. Sergi, C. Stefano, S. Ioppolo, P. Piraino, "Investigations on ancient mortars from the Basilian monastery of Fragal", J. Therm. Anal. Calorim. 91 (2008) 477.

[30] V.R. Zamudio, C.A. Chavez, J. Cervantes, "Clay minerals in historic buildings", J. Therm. Anal. Calorim. 104 (2011) 405.

[31] S. Gunasekaran, G. Anbalagan, "Thermal decomposition of natural dolomite", Bull. Mater. Sci. 30 (2007) 339.

[32] S. Shoval, M. Gaft, P. Beck, Y. Kirsh, "Thermal behavior of limestone and monocrystalline calcite tempers during firing and their use in ancient vessels", J. Therm. Anal. 40 (1993) 263.

[33] S.J.G. Sousa, J.N.F. Holanda, "Development of red wall tiles by the dry process using Brazilian raw materials", Ceram. Int. 31 (2005) 215.

[34] A. Salem, S.H. Jazayeri, E. Rastelli, G. Timellini, "Dilatometric study of shrinkage during sintering process for porcelain stoneware body in presence of nepheline syenite", J. Mater. Process. Technol. 209 (2009) 1240.

[35] A.C.A. Prado, A. Zanardo, M.M.T. Moreno, A.P.M. Menegazzo, "Redução da susceptibilidade à deformação piroplástica das argilas do Pólo Cerâmico de Santa Gertrudes através da adição de matérias-primas", Cerâmica 54 (2008) 7.

[36] R.A.L. Soares, R.J.S. Castro, R.M. Nascimento, "Estudo da potencialidade da aplicação de uma argila contaminada com calcário na produção de placas cerâmicas", Cerâmica 58 (2012) 475.

[37] B.C.A. Pinheiro, A.G.P. da Silva, "Uso de matériasprimas do Rio Grande do Norte na preparação de massa cerâmica para grês porcelanato", Cerâm. Ind. 15 (2010) 29. (Rec. 17/09/2015, Rev. 23/11/2015, Ac. 15/12/2015) 